\title{
Dynamic load balancing policy in grid computing with multi-agent system integration
}

\begin{abstract}
The policy in dynamic load balancing, classification and function are variety based on the focus study for each research. They are different but employing the same strategy to obtain the load balancing. The communication processes between policies are explored within the dynamic load balancing and decentralized approaches. Multi-agent system characteristics and capabilities are explored too. The unique capabilities offered by multi-agent systems can be integrated or combined with the structure of dynamic load balancing to produce a better strategy to produce a better dynamic load balancing algorithm with multi-agent systems.
\end{abstract}

Keyword: Dynamic load balancing; Policy; Multi-agent system; Grid 